\title{
AN INTERNAL VARIABLE FINITE-STRAIN THEORY OF PLASTICITY WITHIN THE FRAMEWORK OF CONVEX ANALYSIS
}

\author{
BY \\ R. A. EVE, T. GÜLTOP, AND B. D. REDDY* \\ University of Cape Town, South Africa
}

\begin{abstract}
An internal variable constitutive theory for elastic-plastic materials undergoing finite strains is presented. The theory is based on a corresponding study in the context of small strains [6], and has the following features: first, with a view to embracing the classical notions of convex yield surfaces and the normality law, the evolution law is developed within the framework of nonsmooth convex analysis, which proves to be a powerful unifying tool; secondly, the special case of elastic materials is recovered from the theory in a natural manner. After presentation of the theory a concrete example is discussed in detail.
\end{abstract}

1. Introduction. The theory of plasticity in its classical small-strain form is wellestablished, especially that form which the theory takes in its application to metals. The finite-strain theory, on the other hand, while showing indications that it is on the way to becoming an established branch of mechanics, is still nevertheless the subject of considerable effort and debate, and certain of its aspects remain unsettled. The literature on the subject has in the meantime acquired voluminous proportions; without attempting a comprehensive survey we mention as important contributions the early work of Green and Naghdi [7] and of Lee [17] in which were addressed, inter alia, the question of the decomposition of deformation into elastic and plastic parts, the former proposing an additive decomposition of strain and the latter a multiplicative decomposition of deformation gradient. The multiplicative decomposition has proved more popular, and forms the basis for a number of alternative theories; as examples we mention the works of Mandel [18], Halphen and Nguyen [8], Simo and Ortiz [30], Simo [29], and of Moran, Ortiz, and Shih [22]. Kim and Oden [14, 15, 16] have, in a series of papers, discussed other forms of decomposition of the deformation. An important alternative, in use for some time now, is the decomposition of deformation rate into elastic and plastic parts [11, 12, 24, 27].

A further key aspect of any theory of plasticity, and in particular of a finite-strain theory, is the form taken by the evolution equation or flow law. One approach, which

Received March 9, 1989.

* Author's mailing address: Department of Applied Mathematics. University of Cape Town, 7700 Rondebosch, South Africa.

(C)1990 Brown University 
for the finite-strain case can be traced back to the works of Mandel [18], Halphen and Nguyen [8], and Rice [27], has been to develop a theory within the framework of an internal variable theory, and to express the evolution equation in terms of a potential. This approach in turn has as its precursors the work of Moreau [23] and of Kestin and Rice [13] for the small-strain case.

Recently a detailed study has been made [6] of evolution laws for elastic-plastic materials undergoing small deformations, with specific attention devoted to those laws expressed in terms of internal variables and their conjugate forces. This study has been carried out within the framework of convex analysis, and apart from recovering the result, known to Halphen and Nguyen [8], which expresses the evolution law as a normality law and dually in terms of a dissipation function (in the language of convex analysis, in terms of the normal cone to a closed convex set and dually in terms of the subdifferential of the support function of that set) the study goes one step further by clarifying the equivalence of these two forms of the flow law with the maximum plastic work inequality. This last result requires the notion of a maximal responsive map, introduced in [6]. Another result presented in [6] shows that there is a unique convex positively homogeneous function, called the canonical yield function, whose level set at unity coincides with the yield surface, and which is the polar conjugate (see $[6,28]$ ) of the dissipation function. Our aim in this contribution is to extend the theory developed in [6] to the finite-strain case, at the same time preserving as far as possible the inherently simple structure of the small-strain theory.

We mention that the work of Kim and Oden [14] exhibits some features which are similar to those which we will present; however, the broader framework which these authors adopt-the mathematical basis for their work is nonsmooth, nonconvex analysis-results in a theory which differs in its thrust in many important respects from that to be presented here. By confining the scope of our work to the framework of nonsmooth convex analysis we are able to derive a variety of results not obtainable in the more general case.

It is worth noting that the recent work by Simo [29] on constitutive relations in finite-strain plasticity is also based on a maximum plastic work inequality. However, this work differs from ours in two important respects: first, in [29] the dissipation is maximised amongst all deformations, holding the internal variable constant, whereas in its original form [9] the dissipation is maximised amongst all stresses. Our theory in fact generalises the conventional form of the inequality by postulating that the dissipation associated with an internal variable rate is maximised amongst all forces conjugate to the internal variable, by the actual such force.

Another work of interest and of relevance to our own is the recent contribution by Antman and Szymczak [1]. These authors use an internal variable approach to develop a constitutive theory for elastic-plastic materials undergoing finite deformations. They then make use of this theory in a numerical investigation of shocks in problems characterised by one spatial dimension. Also of interest in this work is a discussion by the authors of particular classes of problems, the analysis of which represents both a challenge and an opportunity to obtain a deeper understanding of the nature of finite elastoplasticity. 
A significant difference between our work and, in fact, most work in this area, is that we embed the theory in a convex analytic framework from the outset. It is particularly noticeable that while most theories do not do this, the examples used to illustrate such theories invariably make use of convex sets or functions (for example, convex yield surfaces). The rich structure of convex analysis will be seen to be admirably suited to a theory of finite-strain plasticity. While we adopt convexity as basic to our theory, we nevertheless do not impose assumptions of smoothness on the functions, or on the boundaries of sets.

The plan of this work is as follows. In Sec. 2 we summarise results pertaining to the kinematics of elastic-plastic behaviour. In particular we choose to work with a multiplicative decomposition of deformation gradient, and on the basis of this decomposition arrive at an additive decomposition of deformation rate. Then in Sec. 3 we set out the thermodynamic framework in which the internal variable theory is to be developed. Reduced forms of the free energy function, of the stress and of the dissipation inequality are obtained here. In order to construct the evolution law, a number of results concerning convex sets and convex functions are required; these results are presented in Sec. 4 . In Sec. 5 the evolution law is constructed. It generalises the notions of a convex region of admissible stresses and the classical normality law, and is derived by appealing to the form which the dissipation inequality takes, in conjunction with the results from convex analysis. Finally, in Sec. 6 we give a detailed presentation of an example involving an elastic-plastic material which exhibits kinematic and isotropic hardening.

2. Kinematics of elastic-plastic behaviour. As a basis for the description of the kinematics of elastic-plastic behaviour we make use of the multiplicative decomposition of deformation gradient $\mathbf{F}$ [17]: the deformation gradient is written as a composition of an elastic part $\mathbf{F}^{e}$ and a plastic part $\mathbf{F}^{p}$, in the form

$$
\mathbf{F}=\mathbf{F}^{e} \mathbf{F}^{p}
$$

This decomposition is nonunique since for any orthogonal tensor $\mathbf{Q}$ we may write

$$
\mathbf{F}^{e} \mathbf{F}^{p}=\mathbf{F}^{e} \mathbf{Q} \mathbf{Q}^{T} \mathbf{F}^{p}=\mathbf{G}^{e} \mathbf{G}^{p},
$$

say, where $\mathbf{G}^{e}=\mathbf{F}^{e} \mathbf{Q}$ and $\mathbf{G}^{p}=\mathbf{Q}^{T} \mathbf{F}^{p}$ are alternative elastic and plastic parts of F. In any specific application the decomposition may be chosen in a unique way by making specific the choice of $\mathbf{Q}$. For example, according to the polar decomposition theorem $\mathbf{F}^{e}$ and $\mathbf{F}^{p}$ may be decomposed uniquely in the forms

$$
\begin{aligned}
\mathbf{F}^{e} & =\mathbf{R} \mathbf{U}^{e}=\mathbf{V}^{e} \mathbf{R}, \\
\mathbf{F}^{p} & =\mathbf{S U}^{p}=\mathbf{V}^{p} \mathbf{S},
\end{aligned}
$$

where $\mathbf{U}^{e}, \mathbf{V}^{e}, \mathbf{U}^{p}$, and $\mathbf{V}^{p}$ are positive definite symmetric tensors and $\mathbf{R}, \mathbf{S}$ are proper orthogonal tensors representing rotations. The decomposition (1) is rendered unique by choosing, for example, $\mathbf{Q}=\mathbf{R}^{T}$ so that $\mathbf{F}^{e}$ is positive-definite symmetric and all of the rotation is contained in $\mathbf{F}^{p}$. Alternatively, one might choose $\mathbf{Q}=\mathbf{S}$ 
so that $\mathbf{F}^{p}$ instead is positive-definite symmetric. Without specifying any particular choice (this is not required for subsequent developments) we assume that a decomposition has been chosen once and for all.

The decomposition (1) effectively defines a third, intermediate configuration in addition to the reference configuration (which is taken here to coincide with the undeformed, unstressed configuration) and the current configuration, in the sense that $\mathbf{F}^{p}$ may be regarded as a local map of a neighbourhood of a material point in the reference configuration to a corresponding neighbourhood in a configuration which is due solely to plastic flow (Fig. 1). Alternatively, the intermediate configuration of a neighbourhood of a material point may be regarded as the configuration assumed by such a neighbourhood after it has been mapped to its current configuration and then allowed to unload elastically. We emphasise that these interpretations apply locally; in particular, $\mathbf{F}^{p}$ is not the gradient of a motion from the reference to the intermediate configuration (see, for example, [14, 22]).

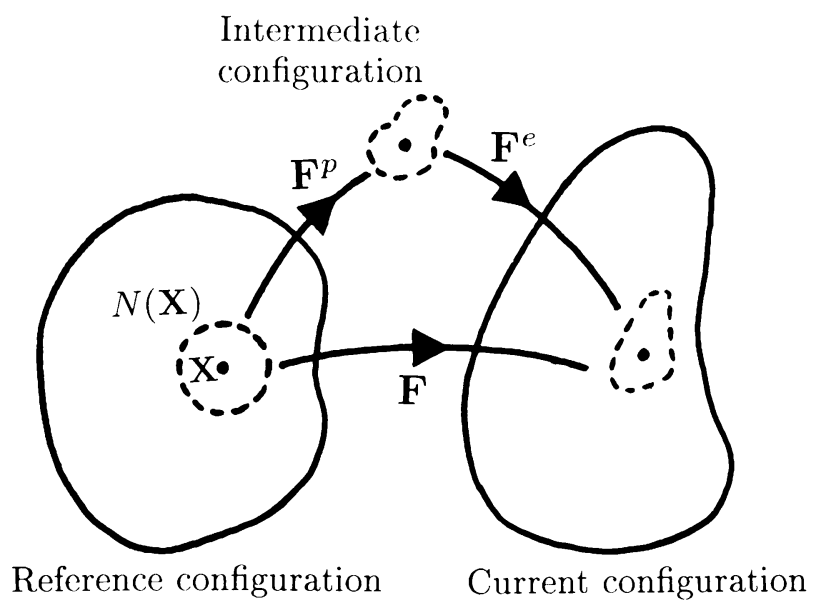

FIG. 1. Reference and current configurations of a material body and the reference, intermediate, and current configurations of a neighbourhood $N(X)$ of a material point $X$.

We take the opportunity to define other kinematic quantities which will be of use later. The Cauchy-Green tensors $\mathbf{C}$ and $\mathbf{B}$ are defined by

$$
\mathbf{C}=\mathbf{F}^{T} \mathbf{F}, \quad \mathbf{B}=\mathbf{F} \mathbf{F}^{T} ;
$$

similarly, we may define elastic and plastic counterparts of these tensors by

$$
\mathbf{C}^{e}=\mathbf{F}^{e T} \mathbf{F}^{e}, \quad \mathbf{B}^{e}=\mathbf{F}^{e} \mathbf{F}^{e T}
$$

and

$$
\mathbf{C}^{p}=\mathbf{F}^{p T} \mathbf{F}^{p}, \quad \mathbf{B}^{p}=\mathbf{F}^{p} \mathbf{F}^{p T} .
$$

The velocity gradient $\mathbf{L}$ is related to the rate of change of deformation gradient by

$$
\mathbf{L}=\dot{\mathbf{F}} \mathbf{F}^{-1} \text {. }
$$


By using (1) and (7) we obtain in a natural way the decomposition of $\mathbf{L}$ into its elastic and plastic parts, that is,

$$
\mathbf{L}=\mathbf{L}^{e}+\mathbf{L}^{p}
$$

where

$$
\mathbf{L}^{e}=\dot{\mathbf{F}}^{e} \mathbf{F}^{p} \mathbf{F}^{-1}, \quad \mathbf{L}^{p}=\mathbf{F}^{e} \dot{\mathbf{F}}^{p} \mathbf{F}^{-1} .
$$

The deformation rate $\mathbf{D}$ and its elastic and plastic parts $\mathbf{D}^{e}$ and $\mathbf{D}^{p}$, respectively, are defined to be the symmetric parts of $\mathbf{L}, \mathbf{L}^{e}$, and $\mathbf{L}^{p}$, that is,

$$
\mathbf{D}=\mathbf{D}^{e}+\mathbf{D}^{p}
$$

where

$$
\mathbf{D}^{e}=\frac{1}{2}\left(\mathbf{L}^{e}+\mathbf{L}^{e T}\right), \quad \mathbf{D}^{p}=\frac{1}{2}\left(\mathbf{L}^{p}+\mathbf{L}^{p T}\right) .
$$

We also have, from (6) and (11),

$$
\dot{\mathbf{C}}^{e}=2 \mathbf{F}^{e T} \mathbf{D}^{e} \mathbf{F}^{e} .
$$

Finally, the assumption that there is no volume change accompanying plastic deformation may be imposed by setting

$$
\operatorname{det} \mathbf{F}^{p}=1,
$$

which in turn implies that

$$
0=\left(\operatorname{det} \mathbf{F}^{p}\right)^{\cdot}=\left(\operatorname{det} \mathbf{F}^{p}\right) \operatorname{tr} \mathbf{L}^{p},
$$

or, with the physically reasonable assumption that $\operatorname{det} \mathbf{F}^{p}>0$,

$$
\operatorname{tr} \mathbf{D}^{p}=0,
$$

where det and $\operatorname{tr}$ denote respectively the determinant and trace of a matrix or second-rank tensor.

3. Constitutive equations. The thermodynamics of deformable media exhibiting dissipative behaviour has as its basis the equation

$$
\rho(\psi+s \theta)^{\cdot}=\overline{\mathbf{T}} \cdot \mathbf{D}-\operatorname{div} \mathbf{q}+\rho r
$$

of balance of energy and the second law of thermodynamics (the Clausius-Duhem inequality)

$$
\rho(\dot{\psi}+s \dot{\theta})-\overline{\mathbf{T}} . \mathbf{D}+\theta^{-1} \mathbf{q} \cdot \nabla \theta \leq 0,
$$

where $\rho$ is the mass density, $\psi$ is the free energy, $\theta$ is the temperature, $\nabla \theta$ the temperature gradient, $s$ the entropy density, $\overline{\mathbf{T}}$ the Cauchy stress, $\mathbf{q}$ the heat flux vector and $r$ the heat supply [5]. A superposed dot denotes material differentiation with respect to time.

We introduce internal variables $\gamma$ and $\mathbf{A}$ which describe hardening behaviour and other effects associated with internal rearrangements of the material. The variable $\gamma$ is a scalar while $\mathbf{A}$ is a second-rank symmetric tensor with respect to the reference configuration. We exclude from consideration internal variables which are vectors since it is known [5] that these would lead to a violation of the principle of material frame indifference. In general there will be an array of both scalar and tensor internal 
variables; however, for simplicity we include one of each kind in the development of the theory. The extension to arrays of internal variables may be accomplished in a straightforward manner.

We are concerned with constitutive theories for inelastic materials, and in order to focus on issues which are central to materials of this type we confine attention to isothermal behaviour, so that $\nabla \theta=\mathbf{0}, \mathbf{q}=\mathbf{0}$, and $r=0$, and $\theta$ is omitted as a variable. Furthermore, for convenience we use as a measure of stress the Kirchhoff stress $\mathbf{T}$, related to Cauchy stress $\overline{\mathbf{T}}$ by

$$
\mathbf{T}=J \overline{\mathbf{T}},
$$

where $J=\operatorname{det} \mathbf{F}=\rho_{0} / \rho, \rho_{0}$ being the referential mass density.

We set

$$
\mathbf{S}=\left(\mathbf{F}^{e}\right)^{-1} \mathbf{T}\left(\mathbf{F}^{e}\right)^{-T}
$$

(that is, $\mathbf{S}$ is a symmetric "Piola-Kirchhoff" stress relative to the intermediate configuration) and consider a set of constitutive relations of the form in which

$$
\{\psi, \mathbf{S}\}
$$

are functions of

$$
\left\{\mathbf{F}^{e}, \gamma, \mathbf{A}\right\} \text {. }
$$

To this set must be added a set of evolution equations, which describe the evolution of inelastic behaviour. Instead of setting out explicitly the form which these equations take, we remark simply that we will seek a set of equations in which rates of change of

$$
\left\{\mathbf{F}^{p}, \hat{\gamma}, \mathbf{A}\right\}
$$

are sought as functions of

$$
\left\{\mathbf{F}^{e}, \hat{\imath}, \mathbf{A}\right\} \text {. }
$$

The reason for this vagueness is that, rather than impose a priori a particular structure, we will deduce an appropriate form for the evolution equation from a reduced form of the dissipation inequality together with assumptions which embody notions of convexity of particular sets and functions (see Sec. 4).

It is worth noting at this stage that in the small-strain theory developed in [6], the free energy and stress are taken to be functions of total strain and internal variables. While we will in most respects follow that theory, it proves to be more judicious in the case of finite strains to assume, as we have done, that the free energy and stress depend on the elastic part of the deformation (that is, on $\mathbf{F}^{c}$ ) and on the internal variables. The small-strain theory with total strain as a variable is viable precisely because of the linearity which pervades this theory, for example, in the elastic part of the constitutive law. It is found, however, that a finite-strain counterpart to this theory which makes use of total deformation (through $\mathbf{F}$ ) is unduly restrictive in its range of applicability.

We now apply the principle of material frame indifference to the set of constitutive equations for $\psi$ and $\mathbf{S}$. A change of frame manifests itself essentially as the 
transformation $\mathbf{F} \rightarrow \mathbf{Q F}$, where $\mathbf{Q}$ is a time-dependent proper orthogonal transformation. From the decomposition (1) it is clear that this amounts to the transformation $\mathbf{F}^{e} \rightarrow \mathbf{Q} \mathbf{F}^{e}$, the component $\mathbf{F}^{p}$ evidently being unchanged by the change of frame. Likewise, both $\gamma$ and $\mathbf{A}$ are left unchanged by the transformation, the first because it is a scalar and the second because it is a referential quantity. Under these circumstances the principle of material frame indifference requires that

$$
\begin{aligned}
& \psi\left(\mathbf{F}^{e}, \gamma, \mathbf{A}\right)=\psi\left(\mathbf{Q F ^ { e }}, \gamma, \mathbf{A}\right), \\
& \mathbf{S}\left(\mathbf{F}^{e}, \gamma, \mathbf{A}\right)=\mathbf{S}\left(\mathbf{Q} \mathbf{F}^{e}, \gamma, \mathbf{A}\right) .
\end{aligned}
$$

By arguing in the usual way [5] we obtain from (17) representations for $\psi$ and $\mathbf{S}$ in the reduced forms

$$
\begin{aligned}
& \psi=\psi\left(\mathbf{C}^{\ell}, \gamma, \mathbf{A}\right), \\
& \mathbf{S}=\mathbf{S}\left(\mathbf{C}^{e}, \gamma, \mathbf{A}\right) .
\end{aligned}
$$

If we define thermodynamic forces $g$ and $\mathbf{G}$ conjugate to $\gamma$ and $\mathbf{A}$, respectively, by

$$
g=-\rho_{0} \frac{\partial \psi}{\partial \gamma}, \quad \mathbf{G}=-\rho_{0} \frac{\partial \psi}{\partial \mathbf{A}}
$$

then substitution of (18) and (19) in the dissipation inequality (14), together with the use of (12), (16) and the assumption of no plastic volume change (Eq. (13)) give the expression

$$
\mathbf{S}=2 \rho_{0} \frac{\partial \psi}{\partial \mathbf{C}^{\rho}}
$$

whence we obtain

$$
\mathbf{T}=2 \rho_{0} \mathbf{F}^{e} \frac{\partial \psi}{\partial \mathbf{C}^{e}} \mathbf{F}^{e T}
$$

for the Kirchhoff stress, and the reduced dissipation inequality

$$
g \dot{\gamma}+\mathbf{G} \cdot \dot{\mathbf{A}}+\operatorname{dev} \mathbf{T} \cdot \mathbf{D}^{p} \geq 0,
$$

where $\operatorname{dev}()$ denotes the deviatoric part of a tensor: $\operatorname{dev} \mathbf{T}=\mathbf{T}-\frac{1}{3}(\operatorname{tr} \mathbf{T}) \mathbf{I}$.

The reduced form (21) of the dissipation inequality will be seen to play a pivotal role later in the construction of an evolution law. Before embarking on a detailed discussion of the form and properties which we intend attributing to this law it is necessary to draw on a number of results from convex analysis, some of them standard and others presented for the first time in [6], in the context of the smallstrain theory. Accordingly we review in the next section those concepts and results required subsequently.

4. Review of results from convex analysis. We assume familiarity with elementary concepts of convex analysis; a good reference is [28], and a review may be found in [6]. We concentrate here on the interrelationships between various sets and functions which are especially relevant to plasticity. Throughout this section, $E$ is a finitedimensional space (isomorphic to $R^{n}$ for appropriate $n$ ) and $K$ is a subset of $E$.

The normal cone $N_{K}(\mathbf{x})$ to a convex set $K$ at $\mathbf{x}$ is a set in $E$ defined by

$$
N_{K}(\mathbf{x})=\{\mathbf{p} \in \mathbf{E}:(\mathbf{y}-\mathbf{x}) . \mathbf{p} \geq 0 \forall \mathbf{y} \in K\}
$$




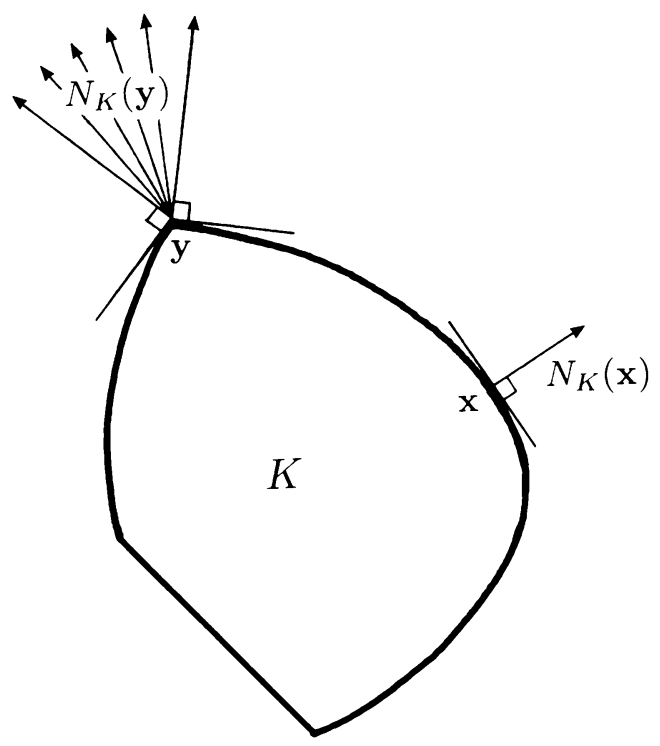

FIG. 2. A convex set $K$ and normal cones $N_{K}(\mathbf{x})$ and $N_{K}(\mathbf{y})$ at a smooth and nonsmooth point, respectively, on the boundary.

(see Fig. 2). From the definition it is clear that for any point $\mathbf{x}$ in the interior of $K$, $N_{K}(\mathbf{x})=\{\mathbf{0}\}$.

The subdifferential $\partial f(\mathbf{x})$ of a convex function $f: E \rightarrow R$ at a point $\mathbf{x}$ is defined to be the set (possibly empty)

$$
\partial f(\mathbf{x})=\{\mathbf{p} \in E: f(\mathbf{y}) \geq f(\mathbf{x})+\mathbf{p} .(\mathbf{y}-\mathbf{x}) \forall \mathbf{y} \in E\}
$$

(see Fig. 3). The elements of $\partial f(\mathbf{x})$ are known as subgradients, and in the event that $f$ is smooth at $\mathbf{x}$ its subdifferential consists of a single member, the gradient $\nabla f(\mathbf{x})$.

A function $f: E \rightarrow R$ is called a gauge if

(i) $f(\mathbf{x}) \geq 0$ for all $\mathbf{x} \in E$;

(ii) $f(\mathbf{0})=0$;

(iii) $f$ is convex, positively homogeneous, and lower semicontinuous (see Fig. 3).

The indicator function $I_{K}$ of $K$ is an extended real-valued function defined on $K$ by

$$
I_{K}(\mathbf{x})= \begin{cases}0 & \text { if } \mathbf{x} \in K, \\ +\infty & \text { otherwise }\end{cases}
$$

and the support function $D_{K}: E \rightarrow R \cup\{+\infty\}$ of $K$ by

$$
D_{K}(\mathbf{p})=\sup _{\mathbf{y} \in K} \mathbf{y} \cdot \mathbf{p} \text {. }
$$

The following result is well-known [28].

Lemma 3.1. Let $K$ be closed and convex. Then

(a) The support function $D_{K}$ is a gauge;

(b) $K=\partial D_{K}(\mathbf{0})$; 


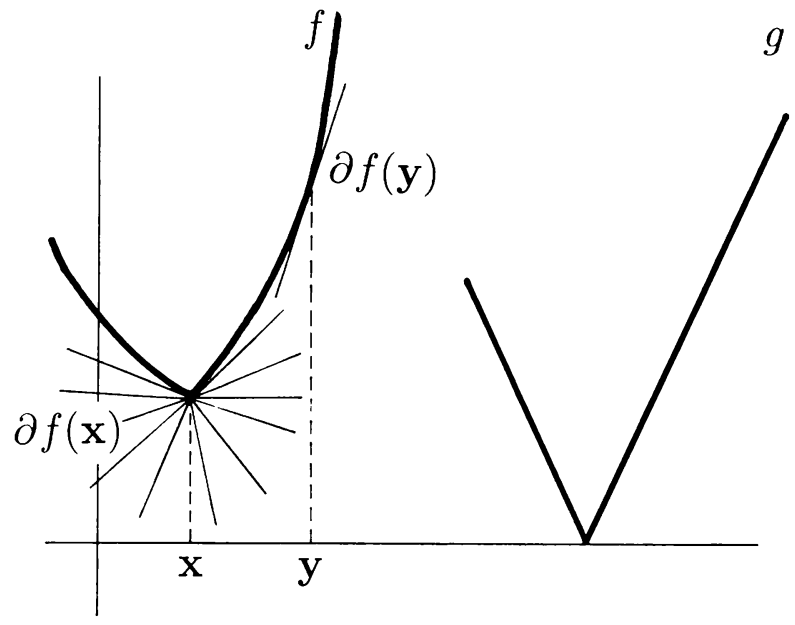

FIG. 3. Examples of a convex function $f$ and its subdifferential, and a gauge $g$.

(c) the subdifferential of the indicator function is the normal cone: $\partial I_{K}=N_{K}$;

(d) if $D_{K}(\mathbf{p})=\mathbf{x . p}$ (that is, the supremum in (25) is achieved at $\mathbf{x}$ ) then

$$
\mathbf{x} \in \partial D_{K}(\mathbf{p}) \quad \text { if and only if } \quad \mathbf{p} \in N_{K}(\mathbf{x}) \text {. }
$$

Remark. Part (d) of the above Lemma is a consequence of the fact that the support and indicator functions of a convex set are conjugate to each other, in the sense of Legendre-Fenchel [28].

In the context of classical small-strain plasticity, if we interpret $K$ as the region of admissible stresses (so that the boundary of $K$ is the yield surface) and $\mathbf{p}$ as the plastic strain rate, then $D_{K}$ is the dissipation function associated with $\mathbf{p}$ and part (d) gives two alternate forms of the evolution equation, the second of which is the normality law. The connection with a yield function is made in the following result $[28,6]$.

Lemma 3.2. Let $D$ be a gauge with the further property that

$$
\mathbf{p} \neq 0 \Rightarrow D(\mathbf{p}) \neq 0 \text {, }
$$

and define the function $f$ on $E$ by

$$
f(\mathbf{x})=\inf \{\mu>0: \mathbf{x} \in \mu K\}
$$

where $K$ is closed and convex, with $\mathbf{0} \in K$. Then

(a) $f$ is a gauge, and

$$
K=\{\mathbf{x}: f(\mathbf{x}) \leq 1\} ;
$$

(b) $f$ and $D$ are polar to each other in the sense that

$$
f(\mathbf{x})=\sup _{\mathbf{p} \neq 0} \frac{\mathbf{x . p}}{D(\mathbf{p})} \quad \text { and } \quad D(\mathbf{p})=\sup _{\mathbf{x} \neq 0} \frac{\mathbf{x . p}}{f(\mathbf{x})} ;
$$

(c) if $\mathbf{x} \in \partial D(\mathbf{p})$ then

$$
\mathbf{x . p}=D(\mathbf{p}) f(\mathbf{x})
$$


(d) if $\mathbf{p} \neq 0$ and $\mathbf{x} \in \partial D(\mathbf{p})$ then there exists a nonnegative scalar $\lambda \geq 0$ such that

$$
\mathbf{p} \in \lambda \partial f(\mathbf{x})
$$

or, if $f$ is smooth,

$$
\mathbf{p}=\lambda \nabla f(\mathbf{x}) \text {. }
$$

Continuing the interpretation with respect to plasticity, we recognise $f$ as the yield function. Given a yield surface, however, there are infinitely many functions $g(\mathbf{x})$, say, whose level surface $g(\mathbf{x})=1$ gives the yield surface. The Lemma identifies one such function $f$ which has the advantage that it is a gauge, and is related to the dissipation function $D$ in the manner described above; we accordingly call the function $f$ the canonical yield function. The above concepts are illustrated in Fig. 4.

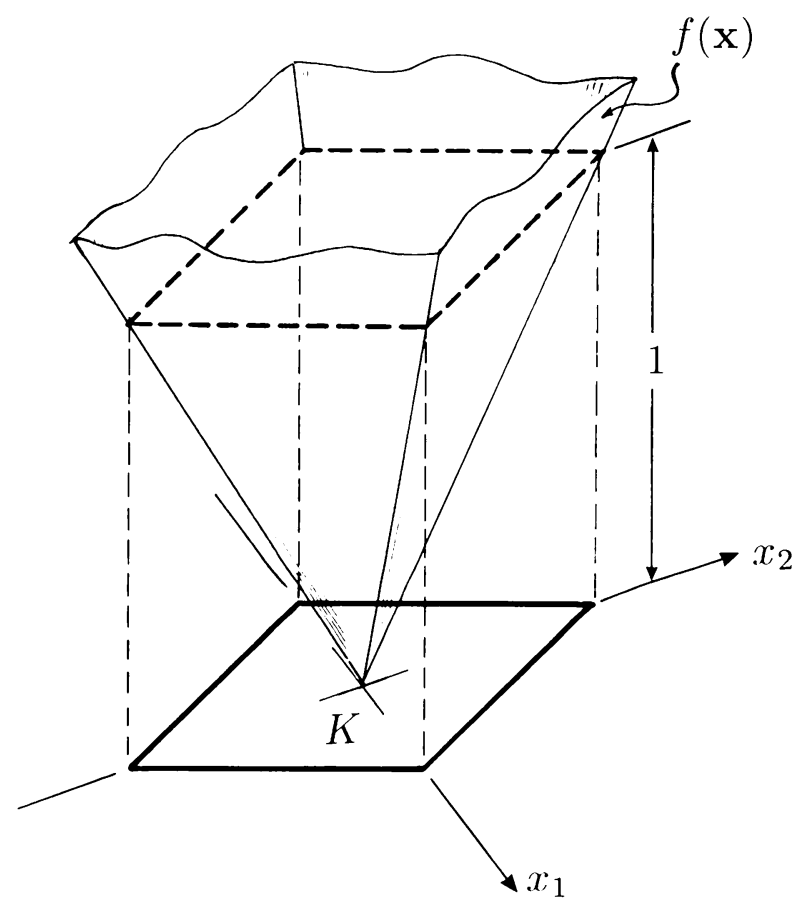

FIG. 4. The construction of a canonical yield function $f$ from the convex set $K$.

The connection with the classical maximum plastic work inequality [9] is made by introducing the notion of a maximal responsive map [6]: a multivalued map $\mathbf{H}: \mathbf{p} \rightarrow \mathbf{H}(\mathbf{p}) \in E$ is said to be responsive if

(i) $\mathbf{0} \in \mathbf{H}(\mathbf{0})$;

(ii) for any $\mathbf{p}_{0}, \mathbf{p}_{1} \in E$,

$$
\left(\mathbf{x}_{0}-\mathbf{x}_{1}\right) \cdot \mathbf{p}_{0} \geq 0 \quad \text { and } \quad\left(\mathbf{x}_{1}-\mathbf{x}_{0}\right) \cdot \mathbf{p}_{1} \geq 0
$$

for all $\mathbf{x}_{0} \in \mathbf{H}\left(\mathbf{p}_{0}\right), \quad \mathbf{x}_{1} \in \mathbf{H}\left(\mathbf{p}_{1}\right)$. 
The map $\mathbf{H}$ is said to be maximal responsive if it is responsive, and if, given $\mathbf{p}_{0}$ and $\mathbf{x}_{0} \in \mathbf{H}\left(\mathbf{p}_{0}\right)$, the inequalities (27) imply that $\mathbf{x}_{1} \in \mathbf{H}\left(\mathbf{p}_{1}\right)$.

The following was proved in [6].

THEOREM 3.1. The set-valued map $\mathbf{H}$ is maximal responsive if and only if there exists a gauge $D$ with the property

$$
\mathbf{H}(\mathbf{p})=\partial D(\mathbf{p}) \quad \forall \mathbf{p} \in E .
$$

Furthermore, when $\mathbf{H}$ is maximal responsive it determines $D$ uniquely and

$$
D(\mathbf{p})= \begin{cases}\mathbf{x} . \mathbf{p} & \text { for all } \mathbf{x} \in \mathbf{H}(\mathbf{p}), \quad \text { if } \mathbf{H}(\mathbf{p}) \neq \varnothing, \\ +\infty & \text { otherwise. }\end{cases}
$$

Lemma 3.1 and Theorem 3.1 allow the evolution equations of classical plasticity to be formulated in three equivalent ways, which are succinctly summarised as follows:

$$
\mathbf{x} \in \mathbf{H}(\mathbf{p}) \Longleftrightarrow \mathbf{x} \in \partial D_{K}(\mathbf{p}) \Longleftrightarrow \mathbf{p} \in N_{K}(\mathbf{x}) ;
$$

here it is understood that $\mathbf{H}$ is maximal responsive, and that $K$ is closed and convex. With the additional assumption (26) we may use Lemma 3.2 to introduce the canonical yield function $f$ which is the polar conjugate to the gauge $D_{K}$.

While such results have been available for some time, their interrelationships have never been made precise (except in a few studies such as those in [23] and [8]) nor has their interpretation within the framework of convex analysis been completely exploited. In particular, while the relationship between the normality law and the dissipation function are-well known [8], the interpretation of the dissipation function and canonical yield function as polar conjugates is new, as is the nature of the equivalence with the maximum plastic work inequality, these having been stated for the first time in [6]. Similar remarks apply to the nature of the equivalence of the maximum plastic work inequality to the notion of a convex yield surface and the normality law; it is Theorem 3.1 which makes this equivalence clear and unambiguous.

5. The evolution equation. We return now to Sec. 2 , with the aim of resolving the issue of the form to be taken by the evolution equation. The intention is to incorporate into this model the classical properties of convexity of yield surfaces and the normality law. Now the groundwork for formalising these ideas has been laid in the previous section, and in order to place our theory in a convex analytic framework it is necessary first of all to decide on the configuration in which to postulate the existence of the appropriate convex sets or functions. Here we are guided by the important observation [10] that, for metal plasticity at any rate, it is the yield function as a function of Cauchy stress which is observed to be convex. It is this notion of convexity which we will incorporate into the structure of the evolution equation, though we will postulate convexity with respect to Kirchhoff stress: since the Kirchhoff and Cauchy stresses differ by a scalar, any set or function which is convex with Cauchy stress as the variable, remains convex with Kirchhoff stress as the variable.

In order to ensure consistency in the arguments which follow, it is necessary that all quantities appearing in the evolution law be spatial in character. Now the relevant quantities are those appearing in the dissipation inequality (21). Of these, $g$ and $\gamma$ 
are scalars, and hence insensitive to the particular description, while $\mathbf{T}$ and $\mathbf{D}^{p}$ are spatial quantities. This leaves the tensorial internal variable $\mathbf{A}$ and its conjugate force $\mathbf{G}$, both of which are referential quantities. The manner in which these two quantities are transformed into spatial quantities will depend on the interpretation given them; for example, A might represent a stress-like or a deformation-like quantity, and its spatial counterpart will differ accordingly. Rather than be unduly prescriptive, we assume that spatial counterparts $\boldsymbol{\Lambda}$ and $\boldsymbol{\Gamma}$ of $\mathbf{A}$ and $\mathbf{G}$, respectively, have been defined, and that these spatial quantities are such that

$$
\mathbf{G} . \dot{\mathbf{A}}=\Gamma . \check{\Lambda},
$$

where $(\checkmark)$ denotes a suitable objective time rate. We now define the array $\mathbf{P}$ by

$$
\mathbf{P}=\left(\mathbf{D}^{p}, \check{\Lambda}, \dot{\gamma}\right)
$$

and a conjugate array $\mathbf{X}$ by

$$
\mathbf{X}=(\operatorname{dev} \mathbf{T}, \boldsymbol{\Gamma}, g)
$$

so that the dissipation inequality $(21)$ becomes

$$
\begin{aligned}
g \dot{\gamma}+\mathbf{G} \cdot \dot{\mathbf{A}}+\operatorname{dev} \mathbf{T} . \mathbf{D}^{p} & =g \dot{\gamma}+\Gamma \cdot \check{\Lambda}+\operatorname{dev} \mathbf{T} . \mathbf{D}^{p} \\
& =\mathbf{X} . \mathbf{P} \\
& \geq 0 .
\end{aligned}
$$

By comparison with the results of Sec. 4 we see that the dissipation inequality together with the notion of a closed convex region of admissible conjugate forces leads in a natural way to an evolution equation which can be postulated in three equivalent forms. Indeed, we assume now that the evolution equation takes the following form:

(i) there exists a closed, convex region $K$ such that $\mathbf{X}$ and $\mathbf{P}$ are related by

$$
\mathbf{P} \in N_{K}(\mathbf{X})
$$

(ii) equivalently,

$$
\mathbf{X} \in \partial D_{K}(\mathbf{p})
$$

(iii) equivalently,

$$
\mathbf{X} \in \mathbf{H}(\mathbf{P})
$$

where $\mathbf{H}$ is a maximal responsive map related to $D$ as in Theorem 3.1.

Furthermore, $D_{K}$ is assumed to possess the property (23), so that there exists a gauge $f$, polar to $D_{K}$, and a scalar $\lambda \geq 0$ such that

$$
\mathbf{P} \in \lambda \partial f(\mathbf{X}) .
$$

The above evolution law is incomplete, since we must add information regarding the skew part of $\mathbf{L}^{p}$, in order to have complete information about the evolution of $\mathbf{F}^{p}$ (see Eq. (9)). Here various options are available, the choice depending to some extent on the particular kind of elastic-plastic material being modelled. We describe two possibilities.

First, we define the plastic spin tensor $\mathbf{W}^{p}$ by

$$
\mathbf{W}^{p}=\frac{1}{2}\left(\mathbf{L}^{p}-\mathbf{L}^{p T}\right) \text {. }
$$


Then one appropriate form of the evolution law for the skew part of $\mathbf{L}^{p}$ is obtained simply by assuming that there is no plastic spin:

$$
\mathbf{W}^{p}=\mathbf{0}
$$

The physical appropriateness and implications of this assumption are discussed by Moran, Ortiz, and Shih [22] and Needleman [24], for example. In this case

$$
\mathbf{D}^{p}=\mathbf{L}^{p}=\mathbf{F}^{e} \dot{\mathbf{F}}^{p} \mathbf{F}^{-1},
$$

whence one may find $\dot{\mathbf{F}}^{p}$, given $\mathbf{D}^{p}, \mathbf{F}$, and $\mathbf{F}^{p}$.

A second option is to assume that $\mathbf{F}^{p}$ is positive-definite and symmetric. Thus in Eqs. (2) and (4) we choose $\mathbf{Q}=\mathbf{S}$ so that

$$
\mathbf{F}^{p}=\mathbf{U}^{p}
$$

where $\mathbf{U}^{p}$ is the positive-definite symmetric part of the polar decomposition of $\mathbf{F}^{p}$, and from (9) and (11) we have

$$
\mathbf{D}^{p}=\operatorname{sym}\left(\mathbf{F}^{e} \dot{\mathbf{U}}^{p} \mathbf{U}^{p-1} \mathbf{F}^{e-1}\right)
$$

so that once again it is possible to determine $\dot{\mathbf{F}}^{p}$ from a knowledge of $\mathbf{D}^{p}$ (when written out in component form relative to any basis, Eq. (38) represents a set of six equations for the six components of $\dot{\mathbf{U}}^{p}$ ).

The material behaviour is thus completely described by the specification of $\psi$ (whence we obtain the relations (19) and (20)), one of $K, \mathbf{H}$, or $D$ (whence we construct the evolution law in one of the forms (33)-(36)), together with an assumption of the form (37) or (38).

An interesting alternative is discussed in [22], where the evolution law is constructed using quantities defined with respect to the intermediate configuration, and where in particular a convex yield function is defined with respect to such quantities. In the case of metals, for which elastic strains are small, there would presumably be little difference between evolution laws which differ only in their use of intermediate rather than spatial quantities as primary variables. We have chosen to make use of spatial variables, but should there be compelling physical evidence for the suitability of intermediate variables, the theory presented here can be modified with little effort, by replacing conjugate pairs of spatial variables with corresponding conjugate intermediate variables.

In problems involving elastic-plastic materials it is found that the evolution equation is most commonly used in the form in which it is expressed as a normality condition (Eq. (33) or (36)). It has been demonstrated [19, 20, 21], however, that there are considerable advantages to using an evolution equation in the form (34) involving the dissipation function, particularly when numerical solutions to the corresponding initial-boundary value problems are sought. The properties of the dissipation function, particularly its convexity and positive homogeneity, allow one to construct rational approximations which in turn place in perspective the well-known schemes for integration of constitutive equations (see [20] for a review). 
6. An example. We consider an example of an elastic-plastic material which exhibits both kinematic as well as isotropic hardening. Kinematic hardening is accounted for by the introduction of an internal variable $\mathbf{A}$ which is assumed symmetric, and isotropic hardening is represented by a scalar $\gamma$, which could be the total plastic work or the effective plastic strain, for example.

We consider a free energy function in the separable form

$$
\psi\left(\mathbf{F}^{e}, \gamma, \mathbf{A}\right)=\psi_{0}\left(\mathbf{F}^{e}\right)+\psi_{1}(\mathbf{A})+\psi_{2}(\gamma),
$$

and proceed to make suitable choices for $\psi_{0}, \psi_{1}$, and $\psi_{2}$. In seeking an appropriate function $\psi_{0}$ we are guided by the notion that when the material is elastic (so that $\mathbf{F}^{e}=\mathbf{F}$ ) the corresponding boundary value problem should be well-posed, in that at least one solution exists in a suitable Sobolev space. While there is as yet no existence theory for elastic-plastic problems with finite strains, it is reasonable to assume that such a theory, based on a free energy of the form (39), would require the function $\psi_{0}$ to have at least the properties which the elastic theory demands, for existence of solutions. Realistic sufficient conditions for this to be the case in elasticity were first given by Ball [2] in respect of the strain energy function (see Ciarlet [3] for an excellent account of this theory). In the present context these conditions amount to the following: it is required that

(a) $\psi_{0}$ be polyconvex, that is, $\psi_{0}$ be expressible as a convex function of $\mathbf{F}^{e}$, cof $\mathbf{F}^{e}$ and $\operatorname{det} \mathbf{F}^{e}$, where cof $\mathbf{A}$ denotes the cofactor of $\mathbf{A}: \operatorname{cof} \mathbf{A}=(\operatorname{det} \mathbf{A}) \mathbf{A}^{-T}$ for invertible $\mathbf{A}$;

(b) $\psi_{0}\left(\mathbf{F}^{e}\right) \rightarrow+\infty$ as $\operatorname{det} \mathbf{F}^{e} \rightarrow 0^{+}$;

(c) $\psi_{0}$ be coercive, in the sense that there exist constants $\alpha, \beta, p, q, r$ such that

$$
\psi_{0}\left(\mathbf{F}^{e}\right) \geq \alpha\left(\left|\mathbf{F}^{e}\right|^{p}+\left|\operatorname{cof} \mathbf{F}^{e}\right|^{q}+\left(\operatorname{det} \mathbf{F}^{e}\right)^{r}\right)+\beta
$$

for all invertible $\mathbf{F}^{e}$, where $\alpha>0, p \geq 2, q \geq \frac{p}{p-1}, r>1$.

For example, we may adopt a free energy function proposed by Ciarlet and Geymonat [4] (see also [3]) which possesses all of the above properties and which has the further merit that it is easily adjustable to experimental results; this function is of the form

$$
\psi_{0}\left(\mathbf{F}^{e}\right)=a\left|\mathbf{F}^{e}\right|^{2}+b\left|\operatorname{cof} \mathbf{F}^{e}\right|^{2}+l\left(\operatorname{det} \mathbf{F}^{e}\right)+e
$$

where $a>0, b>0$, and

$$
l(\delta)=c \delta^{2}-d \log \delta,
$$

with $c>0, d>0$, and $e \in R$. In work which is currently in progress by the authors, this class of free energy functions is being used in numerical studies of finite-strain problems involving elastic-plastic materials, within the framework of the constitutive law developed in this work.

Next, we consider the term $\psi_{1}$ involving the internal variable $\mathbf{A}$. The conjugate force $\mathbf{G}$ is, from (19),

$$
\mathbf{G}=-\rho_{0} \frac{\partial \psi_{1}}{\partial \mathbf{A}} .
$$

We treat $\mathbf{G}$ as a stress-like referential quantity and define its spatial counterpart $\boldsymbol{\Gamma}$ by

$$
\Gamma=\mathbf{F G F}^{T}
$$


so that if the spatial counterpart $\boldsymbol{\Lambda}$ of the internal variable $\mathbf{A}$ is defined by

$$
\mathbf{\Lambda}=\mathbf{F}^{-T} \mathbf{A} \mathbf{F}^{-1} \text {, }
$$

we find that the contribution to the dissipation of this internal variable is, after some manipulation,

$$
\text { G. } \dot{\mathbf{A}}=\boldsymbol{\Gamma} . \check{\Lambda}
$$

where ( $)$ represents the objective time rate defined by

$$
\check{\Lambda}=\dot{\Lambda}+\mathbf{L}^{T} \mathbf{\Lambda}+\mathbf{\Lambda} \mathbf{L} \text {. }
$$

Thus the variable $\check{\Lambda}$ which appears as a component of $\mathbf{P}$ in (30) is defined in a natural way, as an appropriate conjugate quantity.

For consistency with the form which the flow law will take it is necessary also to assume that $\check{\Lambda}$, like $\mathbf{D}^{p}$, is trace-free. Then the dissipation inequality (32) can be written in the form

$$
g \dot{\gamma}+\operatorname{dev} \mathbf{T} . \check{\Lambda}+\operatorname{dev} \mathbf{T} . \mathbf{D}^{p} \geq 0,
$$

with the result that the ordered triples $\mathbf{P}$ and $\mathbf{X}$ take the form

$$
\mathbf{P}=\left(\mathbf{D}^{p}, \check{\Lambda}, \dot{\gamma}\right), \quad \mathbf{X}=(\operatorname{dev} \mathbf{T}, \operatorname{dev} \boldsymbol{\Gamma}, g) .
$$

In order to accommodate nonlinear isotropic hardening we let the internal variable $\gamma$ represent the accumulated plastic work or some scalar measure of accumulated plastic strain, and consider an expression for $\psi_{2}$ of the form

$$
\psi_{2}(\gamma)=\frac{1}{2} c_{1} \gamma^{2}+\left(c_{2}-c_{0}\right)\left(\gamma+\beta^{-1} \exp (-\beta \gamma)\right)
$$

where $c_{0}, c_{1}, c_{2}$, and $\beta$ are material constants with $c_{2}>c_{0}$ (a similar example has been treated in [16]), and of course the force $g$ conjugate to $\gamma$ is given by

$$
g=-\psi_{2}^{\prime}(\gamma)
$$

Finally, we need to specify one of $\mathbf{H}, D$, or $K$. We choose for simplicity a von Mises yield condition, suitably altered to accommodate kinematic and isotropic hardening, so that it is of the form

$$
|\operatorname{dev}(\mathbf{T}+\boldsymbol{\Gamma})| \leq c_{0}+c_{1} \gamma+\left(c_{2}-c_{0}\right)(1-\exp (-\beta \gamma))
$$

where $c_{0}=\sqrt{\frac{2}{3}} T_{0}, T_{0}$ being the yield stress in uniaxial tension. The canonical yield function is thus

$$
f(\mathbf{X})=\frac{|\operatorname{dev}(\mathbf{T}+\boldsymbol{\Gamma})|+g}{c_{0}} .
$$

It is easily established that $f$ is a gauge: it clearly satisfies the conditions $f(\mathbf{X}) \geq$ $0, f(\mathbf{0})=0$, and it is positively homogeneous, so it remains to check lower semicontinuity and convexity. $f$ is in fact continuous, hence lower semicontinuous. Convexity follows from the linearity of $\mathrm{dev}$ and repeated use of the triangle inequality using the norm $|\cdot|$ on $E$.

The flow law (36) now gives

$$
\mathbf{P} \equiv\left(\begin{array}{c}
\mathbf{D}^{p} \\
\check{\Lambda} \\
\dot{\gamma}
\end{array}\right)=\lambda\left(\begin{array}{c}
\mathbf{N} \\
\mathbf{N} \\
1
\end{array}\right)
$$


where the constant $c_{0}$ has been absorbed into $\lambda$, and

$$
\mathbf{N}=\frac{\operatorname{dev}(\mathbf{T}+\boldsymbol{\Gamma})}{|\operatorname{dev}(\mathbf{T}+\boldsymbol{\Gamma})|}
$$

Thus we find that

$$
\dot{\gamma}=\lambda \quad \text { and } \quad \mathbf{D}^{p}=\check{\Lambda} .
$$

In view of (46) and (47) we may restrict attention to the subspace consisting of those vectors $\mathbf{P}$ of the form $\mathbf{P}=(\mathbf{K}, \mathbf{K}, \alpha)$ with $\operatorname{tr} \mathbf{K}=0$, and for vectors in this subspace the dissipation function $D$ is (for simplicity we drop the subscript $K$ on $D$ )

$$
\begin{aligned}
D(\mathbf{P}) & =\sup _{f(\mathbf{X}) \leq 1} \mathbf{X} \cdot \mathbf{P} \\
& =\sup _{f(\mathbf{X})=1} \mathbf{X} \cdot \mathbf{P} \\
& =\sup _{f(\mathbf{X})=1}\left\{\operatorname{dev}(\mathbf{T}+\boldsymbol{\Gamma}) \cdot \mathbf{D}^{p}+g \dot{\gamma}\right\} \\
& \left.=\sup _{f(\mathbf{X})=1}\left\{g\left(\dot{\gamma}-\left|\mathbf{D}^{p}\right| \cos \theta\right)+c_{0}\left|\mathbf{D}^{p}\right| \cos \theta\right)\right\}
\end{aligned}
$$

where

$$
\cos \theta=\frac{(\operatorname{dev}(\mathbf{T}+\boldsymbol{\Gamma})) \cdot \mathbf{D}^{p}}{|\operatorname{dev}(\mathbf{T}+\boldsymbol{\Gamma})|\left|\mathbf{D}^{p}\right|} .
$$

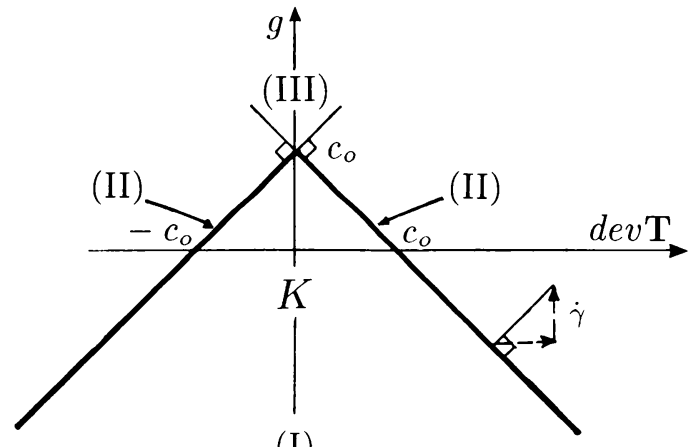

(I)

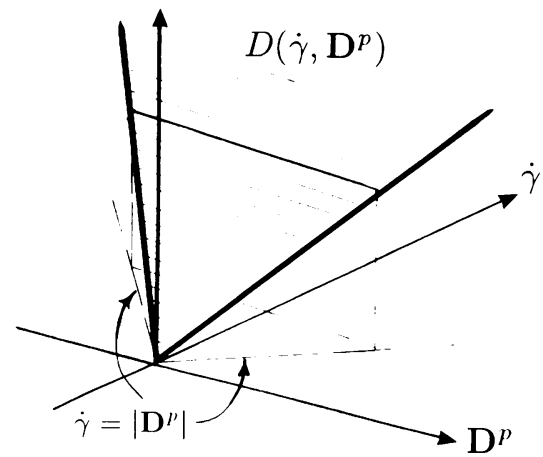

FIG. 5 The region $K$ and the associated dissipation function $D$ for the case in which kinematic hardening is suppressed and $\mathbf{T}$ and $\mathbf{D}^{p}$ are taken to be one-dimensional. 
Three situations are possible: $\dot{\gamma}$ is either less than, equal to, or greater than $\left|\mathbf{D}^{p}\right|$. For each of these situations we evaluate the supremum on the right hand side of (46) to obtain

$$
\begin{aligned}
\text { (I) } \quad & \dot{\gamma}<\left|\mathbf{D}^{p}\right|: D(\mathbf{P}) \rightarrow \infty \\
\text { (II) } \quad & \dot{\gamma}=\left|\mathbf{D}^{p}\right|: D(\mathbf{P})=\dot{\gamma} \\
\text { (III) } \quad & \dot{\gamma}>\left|\mathbf{D}^{p}\right|: D(\mathbf{P})=\dot{\gamma} .
\end{aligned}
$$

The situation is summarised pictorially in Fig. 5, which shows for a one-dimensional situation the canonical yield function and the dissipation function. We note that while the theory gives rise to the three possibilities summarised in (49), in practice it is only (II) which will be possible: the region $g>0$ in the set $K$ is not accessible since we have at all times $\gamma \geq 0 \Longleftrightarrow g \leq 0$, so that case (III) will not arise. It is clear from the normality law and the shape of $K$ that case (I) will not arise either.

7. Concluding remarks. We have constructed a finite-strain theory of plasticity which conforms to the standard requirements for such a theory: it has a sound mathematical and physical basis, and it is simple in structure, hence easy to use. It remains to carry out further detailed studies of the range of specific constitutive laws which are contained in the theory presented here, as special cases. This would amount to the following exercise: given a set of constitutive relations in classical form, define the set of internal variables, the free energy function and one of the yield function, dissipation function, or maximal responsive map which result in the given set of relations being reproduced exactly by our theory. Naturally this will not always be possible; for example, any theory involving a nonconvex potential falls outside the scope of our theory. Nevertheless, it is our contention that the theory presented here provides a framework sufficiently broad in which to model a wide range of materials which are elastic-plastic in nature.

In the case of the small-strain theory the advantages of the framework discussed here are evident in a variety of ways; first, the variational structure of the problem is particularly simple when the evolution law is written in terms of the dissipation function [20], and the properties of this function render the problem well-posed. This may be seen in work presented in [25] and [26]; the former contains an analysis of a problem which has an identical structure to that which is under discussion, while the latter treats a broad class of problems with unilateral constraints, so that the problem being discussed here is a special case. Secondly, it has been demonstrated in $[19,20,21]$ that this same variational framework, when time-discretised in a rational way, gives rise to a problem which in discrete form is amenable to solution by the use of simple algorithms. Naturally it is of great interest to ascertain the extent to which these advantages carry over to the finite-strain case. Certainly one may formulate a variational boundary value problem along the same lines, but the qualitative aspects of such a problem and its approximate solution by numerical means await investigation. These will be discussed in a future contribution.

Acknowledgment. The authors acknowledge the support of the Foundation for Research Development. Part of this work was carried out while B.D.R. was visiting the Institute for Mathematics and its Applications, University of Minnesota. The hospitality of that institution is gratefully acknowledged. 


\section{REFERENCES}

[1] S. S. Antman and W. G. Szymczak, Nonlinear elastoplastic waves, Contemp. Math. 100, 27-54 (1989)

[2] J. M. Ball, Convexity conditions and existence theorems in nonlinear elasticity: Arch. Rational Mech. Anal. 63, 337-406 (1977)

[3] P. G. Ciarlet, Mathematical Elasticity, Volume I: Three-dimensional Elasticity, North-Holland. Amsterdam, 1988

[4] P. G. Ciarlet and G. Geymonat, Sur les lois de comportement en élasticité nonlinéaire compressible. C. R. Acad. Sci. Paris Sér. II 295, 423-426 (1982)

[5] B. D. Coleman and M. E. Gurtin. Thermodynamics with internal state variables. J. Chem. Phys. 47, 597-613 (1967)

[6] R. A. Eve, B. D. Reddy, and R. T. Rockafellar, An internal variable theory of elastoplasticity based on the maximum plastic work inequality, Quart. Appl. Math. 48, 59-83 (1990)

[7] A. E. Green and P. M. Naghdi, A general theory of an elastic-plastic continuum, Arch. Rational Mech. Anal. 18, 251-281 (1965)

[8] B. Halphen and Q. S. Nguyen. Sur les matériaux standards généralisés, J. Méc. 14. 39-63 (1975)

[9] R. Hill, The essential structure of constitutive law's for metal composites and polycrystals, J. Mech. Phys. Solids 15, 79-95 (1967)

[10] R. Hill, On constitutive inequalities for simple materials. II. J. Mech. Phys. Solids 16, 315-322 (1968)

[11] R. Hill and J. R. Rice, Elastic potentials and the structure of inelastic constitutive law's, SIAM J. Appl. Math. 25, 448-461 (1973)

[12] J. W. Hutchinson, Finite strain analysis of elastic-plastic solids and structures, Numerical Solution of Nonlinear Structural Problems (ed. R. F. Hartung), Amer. Soc. Mech. Eng., New York, pp.17291973

[13] J. Kestin and J. R. Rice, Paradoxes in the application of thermodynamics to strained solids. A Critical Review of Thermodynamics (ed. E. B. Stuart), Mono Book Corp.. Baltimore, 1970, pp. 275-298

[14] S. J. Kim and J. T. Oden, Generalized potentials in finite elastoplasticity. Internat. J. Engrg. Sci. 22, 1235-1257 (1984)

[15] S. J. Kim and J. T. Oden. Generalized potentials in finite elastoplasticity, II. Example. Internat. J. Engrg. Sci. 23, 510-530 (1985)

[16] S. J. Kim and J. T. Oden. Finite element analysis of a class of problems in finite elastoplasticity. based on the thermodynamical theory of materials of type $N$. Comput. Methods Appl. Mech. Engrg. 53, 277-302 (1985)

[17] E. H. Lee, Elastic-plastic deformations at finite strains, J. Appl. Mech. 36, 1-6 (1969)

[18] J. Mandel, Thermodynamics and plasticity. Foundations of Continuum Thermodynamics (ed. J. J. Delgado Domingos, M. N. R. Nina, and J. H. Whitelaw), Macmillan, 1974, pp. 283-304

[19] J. B. Martin, An internal variable approach to the formulation of finite element problems in plasticity, Physical Nonlinearities in Structural Analysis (ed. J. Hult and J. Lemaitre), Springer, Berlin. 1981, pp. 165-176

[20] J. B. Martin and B. D. Reddy, Variational principles and solution algorithms for internal variable formulations of problems in plasticity, Omaggio a Giulio Ceradini: Note Scientifiche in Occasione del $70^{\circ}$ Compleanno (ed. U. Andreaus et al.). Università di Roma 'La Sapienza'. Roma, 1988 , pp. $465-477$

[21] J. B. Martin, B. D. Reddy, T. B. Griffin, and W. W. Bird, Application of mathematical programming concepts to incremental clastic-plastic analysis. Engrg. Struct. 9. 171-176 (1987)

[22] B. Moran, M. Ortiz, and C. F. Shih, Formulation of implicit finite element methods for multiplicative finite deformation plasticity. Technical Report. Division of Engineering. Brown Univ.. 1989

[23] J. J. Moreau, Sur les lois de frottement, de viscosité et plasticité. C. R. Acad. Sci 271, 608-611 (1970)

[24] A. Needleman. On finite element formulations for large elastic-plastic deformations, Comp. Struct. 20, 247-257 (1985)

[25] B. D. Reddy and T. B. Griffin, l'ariational principles and convergence of finite element approximations of a holonomic elastic-plastic problem. Numer. Math. 52. 101-117 (1988) 
[26] B. D. Reddy and F. Tomarelli, The obstacle problem for an elastic-plastic body, Appl. Math. Optim. 21, 89-110 (1990)

[27] J. R. Rice, Inelastic constitutive relations for solids: an internal variable theory and its application to metal plasticity, J. Mech. Phys. Solids 19, 433-455 (1971)

[28] R. T. Rockafellar, Convex Analysis, Princeton University Press, New Jersey, 1970

[29] J. C. Simo, A framework for finite strain elastoplasticity based on maximum plastic dissipation and the multiplicative decomposition: Part I. Continuum formulation, Comput. Methods Appl. Mech. Engrg. 66, 199-219 (1988)

[30] J. C. Simo and M. Ortiz, A unified approach to finite deformation elastoplasticity based on the use of hyperelastic constitutive equations, Comput. Methods Appl. Mech. Engrg. 49, 222-235 (1985) 